\title{
Percretismo placentario: manejo con oclusión de arterias ilíacas internas, embolización de arterias uterinas y oclusión de arterias ilíacas comunes
}

\author{
Jorge Hasbun $H^{1}{ }^{1}$, Patricio Palavecino ${ }^{2}{ }^{2}$, Raúl Osorio $P^{1}$, Cesar Moral B. ${ }^{1}$, \\ Claudia Morales H. ${ }^{3}$, Cristián Palma C. ${ }^{4}$, Mario Salamanca Z. ${ }^{1}$ \\ ${ }^{1}$ Unidad de Medicina Materno-Fetal, Departamento de Obstetricia y Ginecología. ${ }^{2}$ Departamento de Radiología e Imá- \\ genes. ${ }^{3}$ Departamento de Anatomía Patológica. ${ }^{4}$ Departamento de Urología. Hospital Clínico de la Universidad de Chile.
}

\section{RESUMEN}

Presentamos el caso de una embarazada con placenta ístmica, percreta, previa central, con extensas anastomosis vasculares y flujo de alta velocidad. En la resolución mediante cesárea-histerectomía, para prevenir la hemorragia intraoperatoria, se utilizó una intervención radiológica en secuencia de oclusión temporal de las ilíacas internas, embolización de las arterias uterinas, reposicionamiento e insuflación de balones en ilíacas comunes. Se logró mantener estabilidad hemodinámica y el control del sangrado; estando ocluida la circulación uterina hubo pérdidas moderadas en la parte final de la operación. Se discuten la característica poco común de la implantación y percretismo ístmico, y el beneficio del uso combinado de la oclusión vascular con la cirugía expedita para minimizar el riesgo materno.

\section{PALABRAS CLAVES: Placenta previa percreta, embolización arterias uterinas, hemorragia obstétrica, radiología vascular intervencional}

\section{SUMMARY}

We present a patient with placenta previa percreta and oclusive. It had extensive vascular anastomosis with high velocity flow, in which resolution by cesarean-hysterectomy was done for bleeding control and followed by temporal occlusion of iliac arteries, embolization of uterine arteries, reposition and insuflation of balloons in common iliac arteries. The patient was stable and moderated bleeding happened to the end of intervention when the uterine arterial circulation was occluded. We discuss the uncommon of isthmic implantation and percretism and the benefits of both vascular occlusion and expedite surgery for to minimize maternal compromise or damage.

\section{KEY WORDS: Placenta previa percreta, uterine artery embolization, obstetric hemorrhage, vascular interventional radiology}

\section{INTRODUCCIÓN}

La cesárea-histerectomía programada ha contribuido a reducir la mortalidad materna por hemorragia obstétrica en los casos de riesgo, sin embargo la intervención se asocia a morbilidad materna grave, riesgo de infección, daño de órganos vecinos y reintervenciones $(1,2)$, lo que ha estimulado el desarrollo de técnicas complementarias para mejorar su eficiencia. 
En placenta acreta (PA) se han descrito pérdidas sanguíneas promedio de $12.140 \pm 8.343 \mathrm{ml}$ en percretismo y $3.630 \pm 2.216 \mathrm{ml}$ en incretismo (3). Para disminuir la hemorragia actualmente se utiliza la cesárea fúndica, seguida de histerectomía total o subtotal, sin remoción placentaria, y el sangrado intraoperatorio se ha controlado por oclusión vascular extraluminal (sutura o pinzamiento) o intravascular (oclusión por balones) (4).

La radiología intervencional ha aportado el uso de catéteres intraarteriales con balones de oclusión colocados en el preoperatorio en ambas arterias ilíacas internas (All) e insuflados después del nacimiento y ligadura del cordón umbilical para reducir la hemorragia, los que se retiran ya confirmada la hemostasia definitiva (5).

Este procedimiento disminuye la hemorragia intraoperatoria, facilitando una intervención eficiente, con menos complicaciones (6) y evita la oclusión extraluminal (ligadura de hipogástricas o pinzamiento de la aorta abdominal). Los resultados con esta técnica han sido alentadores, sin embargo se han descrito algunos casos de fracaso $(7,8)$, siendo un factor a considerar la presencia de una red anastomótica distal (entre los sistemas arteriales vaginal y uterino), con flujo desde la vagina hacia el útero y que no depende directa o exclusivamente de las All $(9,10,11)$. Estas anastomosis, no serían bloqueadas con las técnicas habituales aún correctamente aplicadas, explicando sus fracasos más o menos frecuentes y la recurrencia de la hemorragia $(2,6,7,12)$.

Presentamos el caso clínico de un embarazo ístmico que demostró tempranamente imágenes de acretismo Grado $3(13,14)$, con lagunas placentarias numerosas y desarrollo vascular subplacentario, de flujo turbulento y alta velocidad en dirección a los parametrios, de extensión progresiva con la edad gestacional, asociada a dolor en hipogastrio con sospecha inminente de rotura uterina.

El extenso desarrollo vascular, de magnitud no observada en nuestra experiencia previa, fue interpretado como una red anastomótica con mayor potencial de hemorragia, eventualmente no controlable con los procedimientos habituales y riesgo materno grave (near miss).

Con el objetivo de controlar mejor la hemorragia intraoperatoria y prevenir una pérdida sanguínea masiva, se diseñó una estrategia mejorada en que la cesárea-histerectomía, protegida con la oclusión inicial de ambas All mediante balones, se agregó como modificación, la embolización supraselectiva de las arterias uterinas (AU). Luego, en secuencia, se efectuó el reposicionamiento de los balones desde las All a ambas ilíacas primitivas (AIP) (15) y su insuflación para bloquear un flujo sanguíneo anastomótico distal, eventualmente indiferente a la oclusión de All. Esta intervención radiológica se aplicó durante la fase inicial de la histerectomía antes de la sutura de los pedículos vasculares uterinos.

\section{Caso clínico}

Gestante de 30 años, gesta 3, para 1, con antecedente de cesárea anterior y un aborto espontáneo. En la novena semana de gestación se observó implantación ístmica con desarrollo vascular subplacentario y posteriormente se confirmaronnumerosas lagunas vasculares con flujo turbulento de alta velocidad y placenta previa central percreta (Figuras 1, 2 y 3).

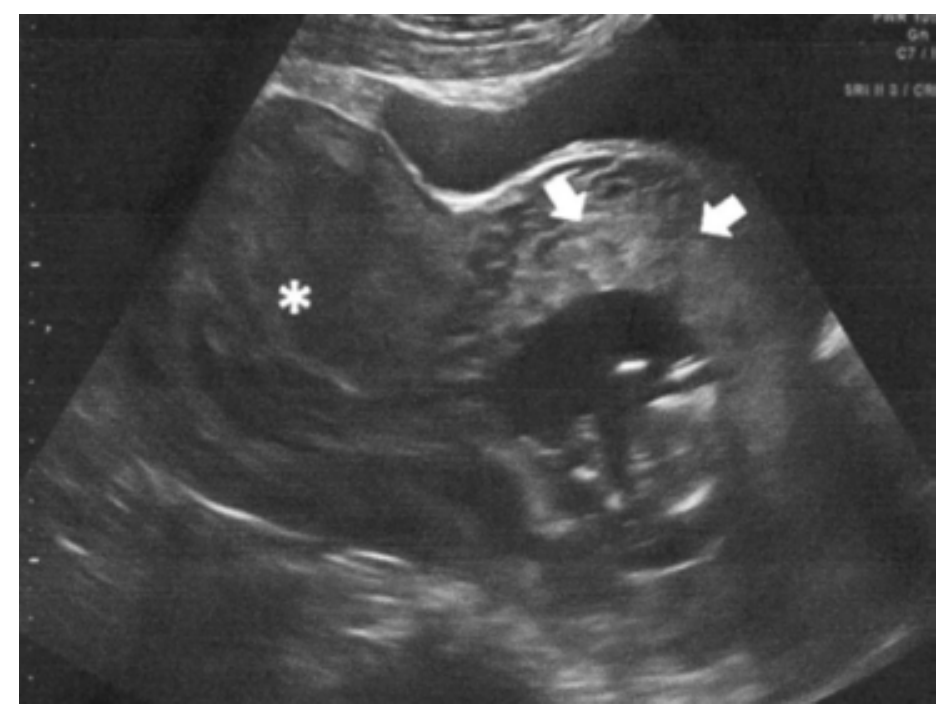

Figura 1. Embarazo en semana 8: se observa imagen uterina abalonada o en "guitarra", implantación ovular ístmica y cavidad uterina del fondo vacía. 


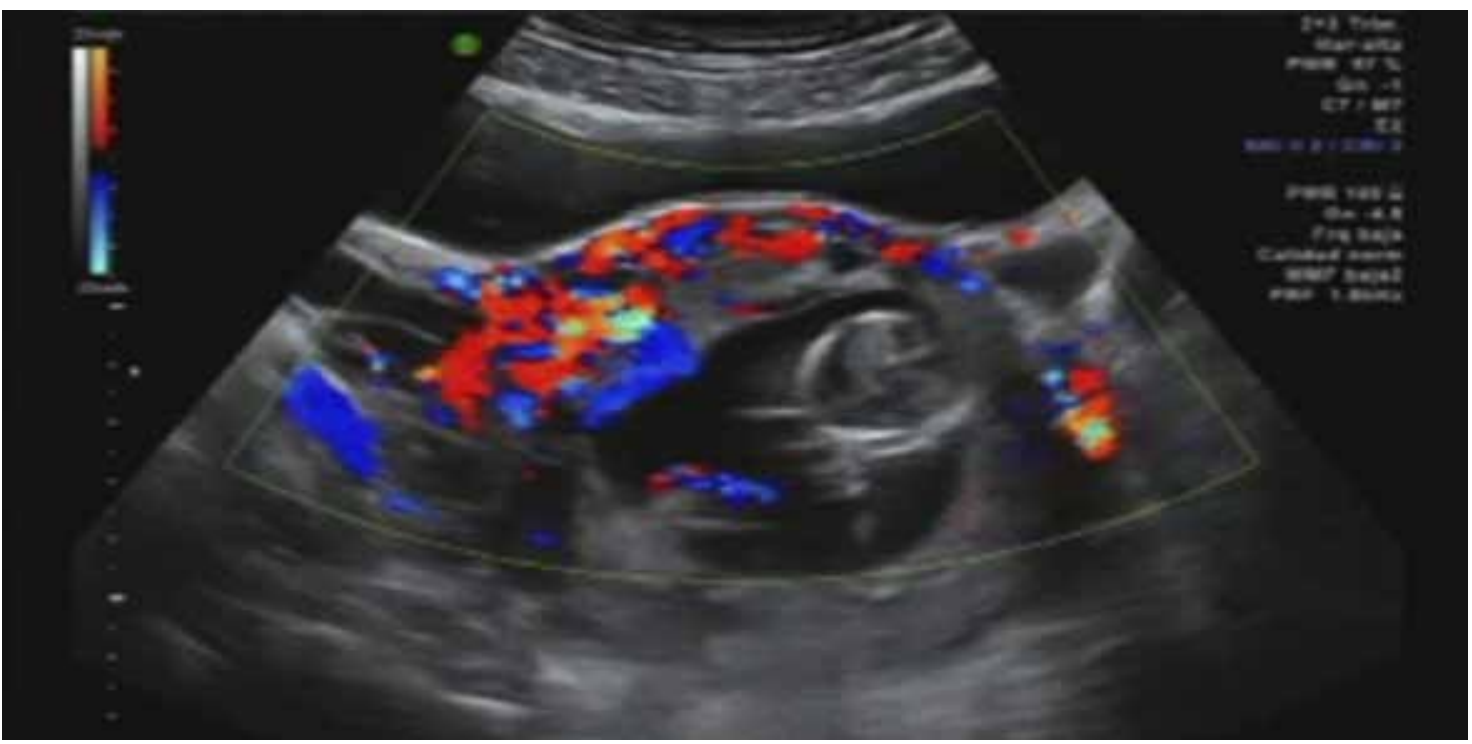

Figura 2. Embarazo ístmico en semana 9 con extensa vascularización anormal bajo el trofoblasto.

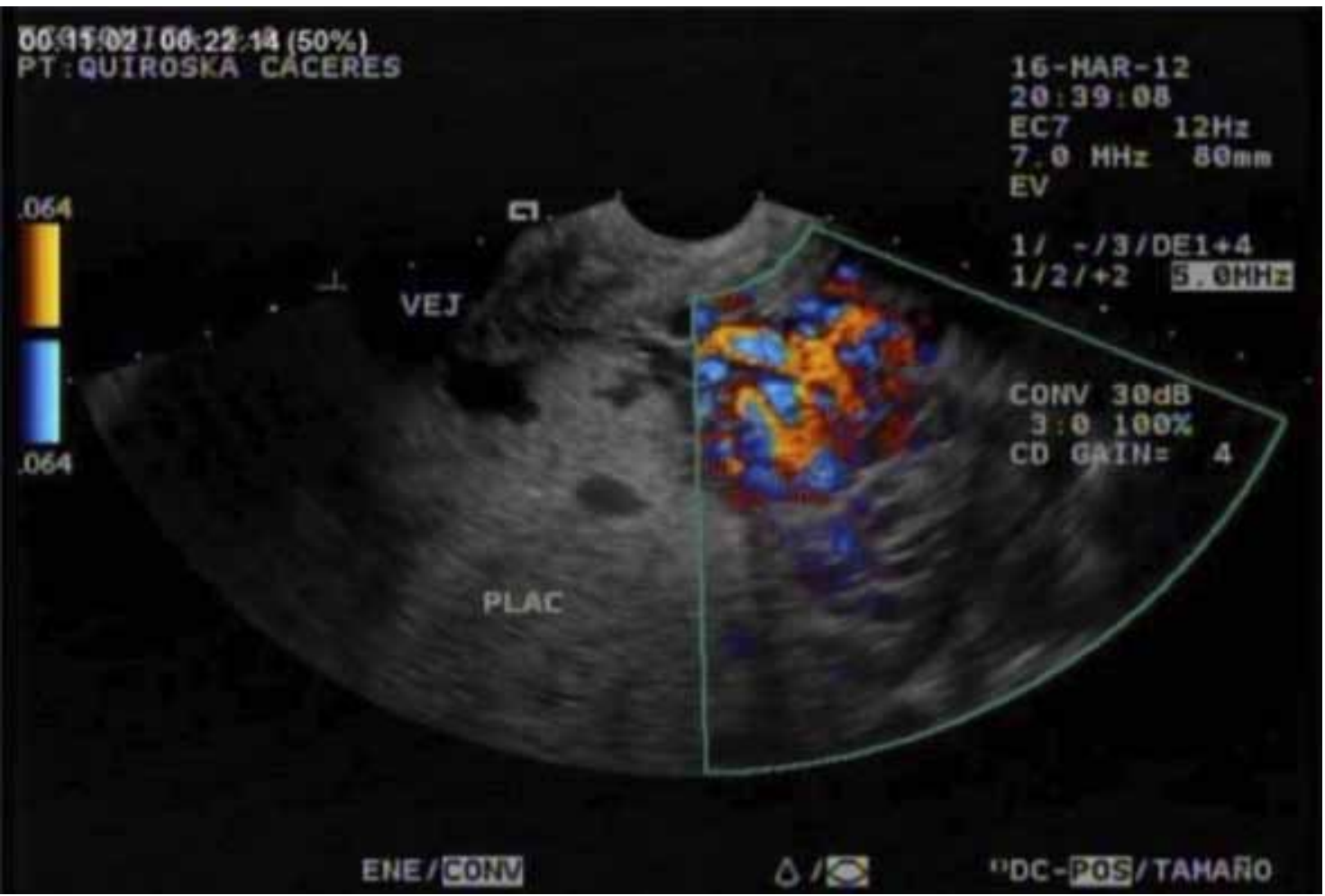

Figura 3. Se observa placenta previa con lagunas vasculares numerosas y proyección anormal de vasos hacia parametrio. 
El crecimiento fetal fue adecuado y la evolución del embarazo sin incidentes agudos, destacando la magnitud de la vascularización en las imágenes y la aparición progresiva desde las 27 semanas, de dolor hipogástrico punzante, mal tolerado y que cedía parcialmente a analgésicos. Esto determinó la hospitalización preventiva en la semana 32 y la interrupción del embarazo por incremento del dolor en la semana 34. La estrategia del manejo perioperatorio fue:

a. Evaluar invasión vesical o rectal (citoscopia y rectoscopia). rales.

b. Colocación preoperatoria de catéteres urete-

c. Instalación preoperatoria de catéteres con balones en All vía femoral.

d. Inicio de cesárea con protocolos diseñados para cirugía y anestesia de PA.

e. Insuflación de balones en All después del nacimiento.

f. Histerorrafia con placenta in situ e inicio de histerectomía con disección de ligamentos anchos, de los pedículos vasculares y detención de la intervención.

g. Embolización de AU.

h. Retiro de catéteres desde AU y reposicionamiento en AIP bajo visión fluoroscópica (arco $\mathrm{C}$ ).

i. Insuflación de balones en AIP.

j. Reinicio y conclusión de histerectomía total.

En el preoperatorio, en sala de angiografía, con anestesia epidural y bajo visión ecográfica con técnica de Seldinger se puncionó ambas arterias femorales comunes instalando balones de oclusión selectivamente en la división anterior de ambas All y se trasladó a pabellón de maternidad.

Con laparotomía media y prolongación paraumbilical de $4 \mathrm{~cm}$, se exteriorizó útero, histerotomía fúndica anteroposterior, extracción de feto en nalgas, ligadura funicular y se insuflan balones de oclusión con $1 \mathrm{ml}$ de solución fisiológica y contraste yodado. Terminada la histerorrafia (Figura 4) se embolizan ambas AU con Gelfoam controlando con pulsos de radioscopias proporcionados por el arco C hasta lograr supresión bilateral del flujo (embolización completa); a continuación se reposicionan los catéteres y se inflan los balones en ambas AIP (Figura 5).

El procedimiento, efectuado por el radiólogo intervencionista, fue expedito, duró 20 minutos y los balones fueron retirados poscirugía, previa observación de hemostasia completa.

Durante la cesárea-histerectomía no hubo pérdida sanguínea importante ni dificultades técnicas; llamó la atención la gran distensión inferior del segmento e istmo, por debajo de los pedículos vasculares ya ligados y con extensión de vasos

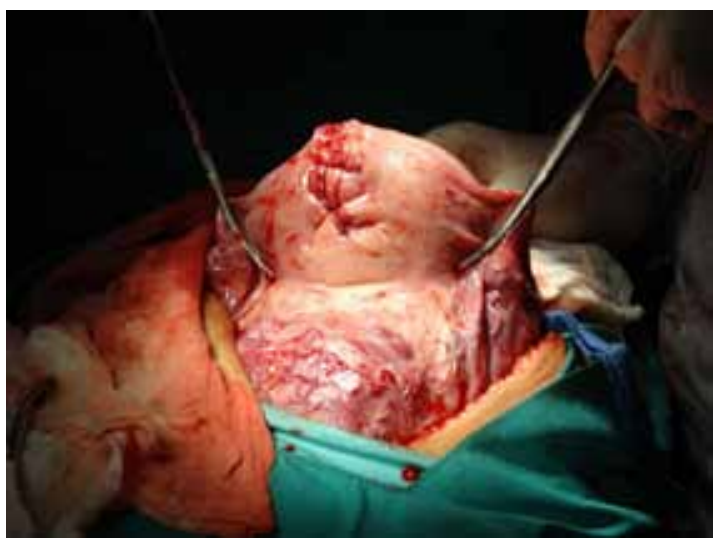

Figura 4. Inicio de histerectomía: se observa cesárea fúndica e histerorrafia antero-posterior, con placenta in situ, previa, percreta.

sanguíneos a paracolpos, que requirió sección y hemostasia desde lateral a línea media, quedando incluido el cuello uterino y $2 \mathrm{~cm}$ de vagina proximal. La placenta previa central se observó percreta ístmica extensa con signos sugerentes de inminencia de rotura uterina (Figuras 6 y 7 ).

La hemodinamia materna se mantuvo estable y el tiempo operatorio fue de 120 minutos. La pérdida sanguínea evaluada fue de $2.500 \mathrm{ml}$ y el aporte de volumen (relación cristaloides/hemorragia: 3/1) fue de 5.000cc (Ringer lactato $2500 \mathrm{ml}$, Ringer 1000 $\mathrm{ml}$, coloides $1000 \mathrm{ml}$ y glóbulos rojos 2 unidades, $500 \mathrm{ml}$ ) con hematocrito de $31 \%$ al egreso de pabellón.

El postoperatorio fue sin incidentes (primer día en la $\mathrm{UCl}$ ), con retiro de los catéteres ureterales a las 36 horas, comienzo de lactancia natural y recuperación rápida (un proceso inflamatorio menor de la cúpula vaginal remitió con antibioticoterapia).

El recién nacido pesó 2.100 gramos, Apgar 9-9, adecuado para la edad gestacional y evolucionó sin complicaciones.

\section{DISCUSIÓN}

Acretismo: Fue un diagnóstico temprano en el primer trimestre, con inserción ístmica total del trofoblasto, llamando la atención los siguientes hechos:

a) La cavidad corporal uterina se mantuvo vacía y el crecimiento placentario focalizado en el segmento. Evolucionó con una expansión circunferencial, dando al útero una "forma de guitarra" que se mantuvo hasta el parto, con distensión máxima e inminencia de rotura uterina espontánea (Figura 7). 


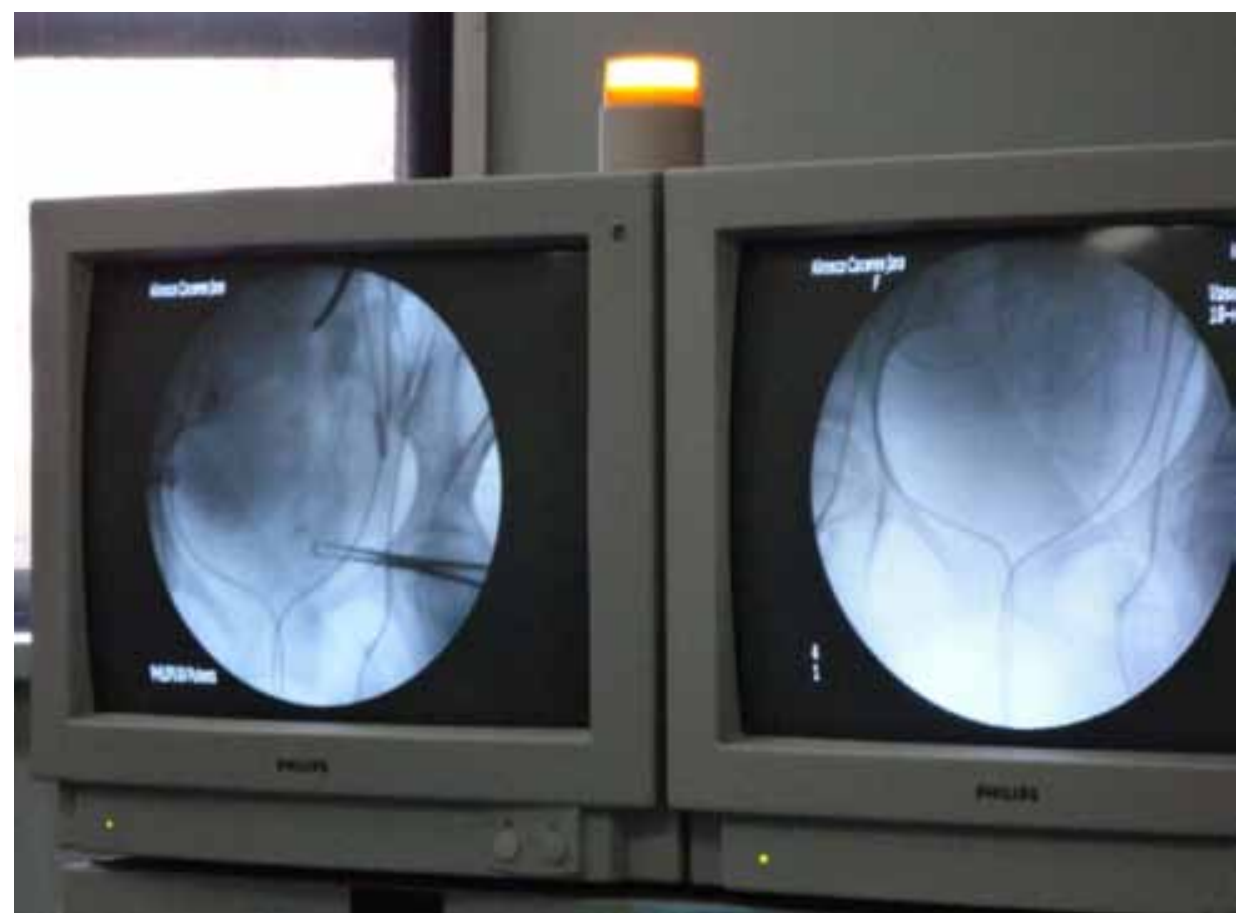

Figura 5. Se observan balones insuflados en la proyección de arterias ilíacas primitivas. En el campo operatorio se visualizan pinzas, compresas y materiales quirúrgicos.

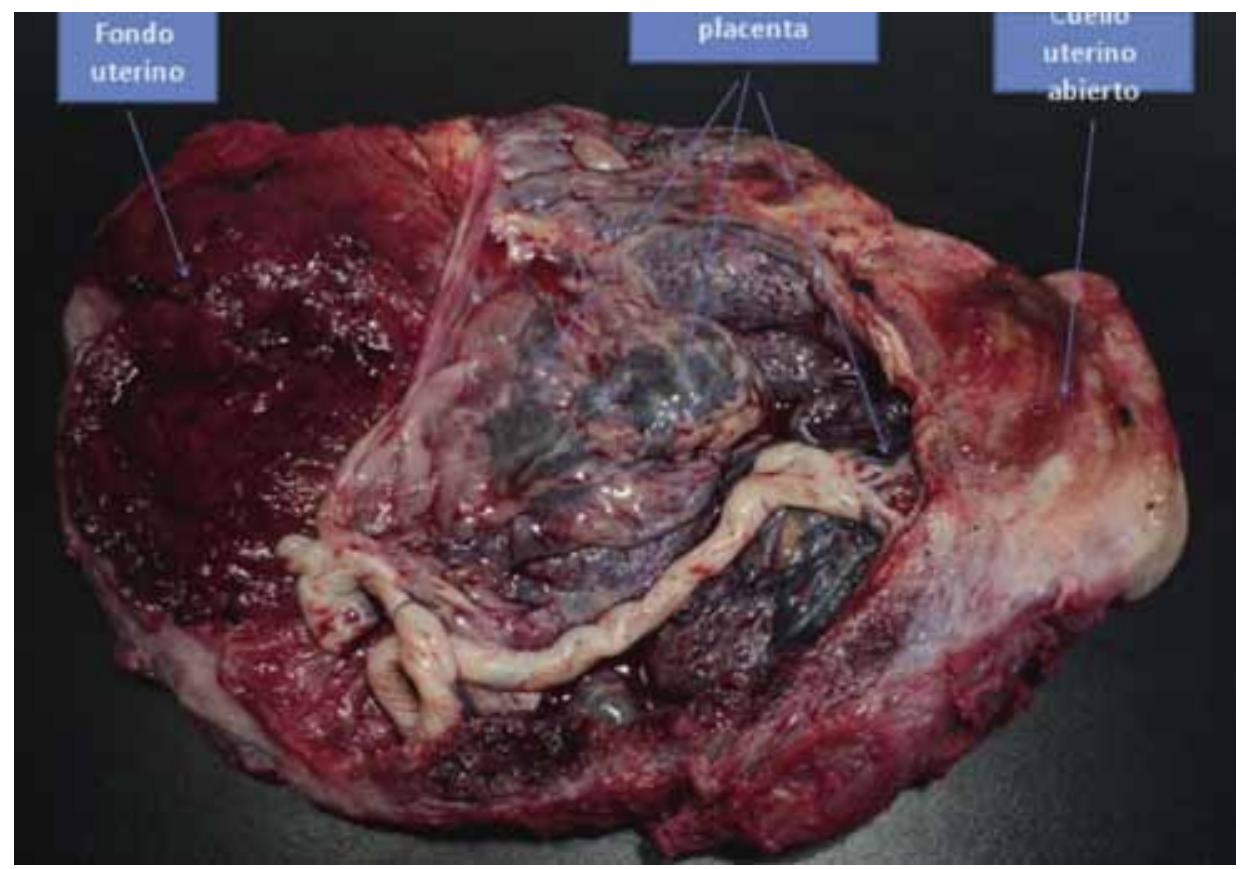

Figura 6. Sección longitudinal de útero con placenta previa que compromete todo el espesor de la pared miometrial. 


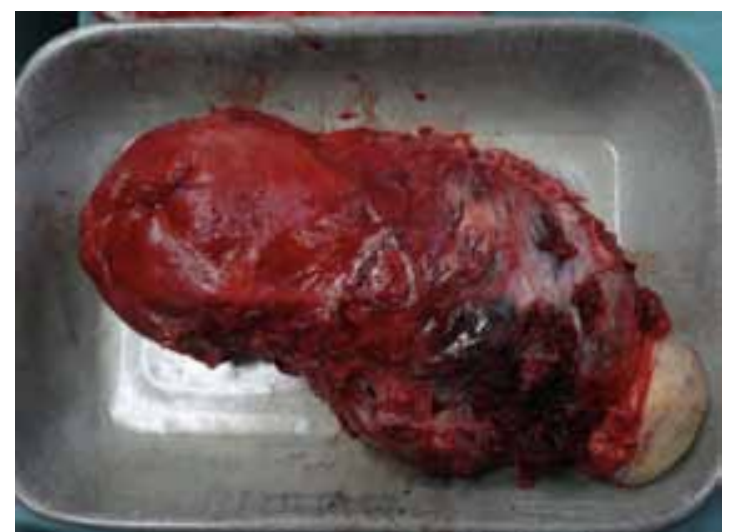

Figura 7. Pieza operatoria de cesárea-histerectomía: útero con placenta in situ, ístmica, percreta, con signos de rotura inminente. Forma uterina evoca imagen ultrasónica de la Figura 1.

b) No hubo migración placentaria ascendente ni percretismo a vejiga o cicatriz uterina, ni metrorragia antenatal, pese a las contracciones de Braxton y a la posición previa central, hecho infrecuente y que representa una adherencia placentaria selectiva, más firme y profunda. La migración, invasión y orientación de la penetración del trofoblasto está regulada por una variedad de factores (proteínas vasoactivas, citoquinas, proteasas, factores de crecimiento y otras) (16) y por la presión parcial de oxígeno tisular que influye en su expresión (17). En placenta previa es frecuente una invasión trofoblástica excesiva asociada a factores de riesgo conocidos (cesárea anterior, raspados, edad avanzada, tabaquismo, drogas, embarazos múltiples). En la cicatrización por cesárea o raspado, hay una secuela de vascularización anormal con hipoxia localizada, determinante de una decidualización defectuosa con invasión trofoblástica en profundidad y adherencia anormal del blastoquiste al tejido cicatricial. Otro mecanismo de penetración descubierto es una diferenciación celular anormal del trofoblasto que adquiere un fenotipo invasor (transición epitelio-mesenquimática) propio de las células tumorales (cambian su forma, pierden polaridad, aumentan su motilidad) (18). En el caso presentado, con antecedente de un raspado uterino por aborto previo, este procedimiento pudo comprometer con mayor fibrosis una cicatriz ístmica muy baja de la cesárea anterior, explicando la ubicación actual de la placenta ya que en el estudio histológico randomizado de pared uterina del segmento no se identificó la cicatriz antigua.

c) El desarrollo vascular subplacentario de las imágenes fue evaluado subjetivamente como "extenso o muy extenso", porque es el mayor obser- vado en nuestra experiencia, estuvo localizado en la mitad inferior del útero, con expansión notable a ambos parametrios y con flujo de alta velocidad, sugiriendo percretismo ístmico. Estudios en arteriografías y en cadáveres $(9,10)$ demuestran que la irrigación más baja o inferior del útero es abastecida por 3 pedículos principales: el superior, que depende de la arteria uterina, el medio que depende de la arteria cervical y el pedículo inferior que recibe sangre de las arterias vaginales, existiendo anastomosis variadas y conexiones ístmicovaginales mediales y en bordes. En este caso, la ubicación placentaria y las imágenes sugerían este tipo de anastomosis requiriendo un área mayor de oclusión vascular y se planificó la intervención radiológica en la secuencia descrita según la etapa quirúrgica. La oclusión lograda se interpretó como eficiente por la hemodinamia materna estable y mejor control de la pérdida sanguínea con volumen total de $2.500 \mathrm{ml}$, inferior al promedio descrito (3), y evitó la necesidad del pinzamiento de la aorta (19). Sin embargo, esta pérdida observada se inició en la etapa final de la histerectomía, con las AU embolizadas, los pedículos vasculares ligados y las AIP ocluidas, indicando el gran potencial hemorrágico de las extensas anastomosis y que fue atenuado por los procedimientos aplicados.

Intervención radiológica: La técnica utilizada es relativamente simple y como otras es dependiente de la experiencia del operador. Tiene una baja tasa de complicaciones y su costo no es tan alto. El caso demuestra que la planificación quirúrgica y radiológica de la intervención evita las dificultades de la improvisación (materiales inadecuados por carencia en stock de urgencia, mesa quirúrgica inapropiada y otros) y favorece el resultado exitoso.

Las diferentes técnicas utilizadas en el manejo de la hemorragia obstétrica han demostrado resultados variables que no parecen deberse a la superioridad de una sobre otra (20), sino que dependen de otros factores como la experiencia de los operadores (cirujanos y radiólogo intervencionista), la oportunidad del procedimiento (programado o de urgencia) y en la paciente misma, de la extensión del acretismo (localización, profundidad e invasividad) evaluado con US, Doppler y eventualmente resonancia.

La evaluación cuidadosa de estos factores en cada caso y la planificación con equipo multidisciplinario pueden reducir el riesgo materno y mejorar los resultados.

Creemos que la expresión observada del acretismo es infrecuente, requiere diagnóstico antenatal oportuno y una adecuada planificación quirúrgica en la interrupción del embarazo. Resumimos el caso clínico en los siguientes puntos:

1. Un caso de percretismo ístmico fue diagnosticado precozmente con el uso de las imágenes.

2. Se detectó un extenso desarrollo vascular en 
parametrios de gran potencial hemorrágico e inminencia de rotura uterina.

3. Para el control hemostático optimizado se efectuó cesárea-histerectomía planificada con secuencia de intervención radiológica: oclusión All, embolización de AU y oclusión de AIP.

4. El volumen de pérdida fue $2.500 \mathrm{ml}$, con estabilidad hemodinámica y hemostasia satisfactoria.

5. No hubo complicaciones con los procedimientos quirúrgico y radiológico.

6. El manejo combinado quirúrgico y radiológico utilizado es recomendable en este tipo de casos.

7. El estudio histopatológico de la pieza operatoria confirmó la interpretación antenatal de las imágenes y la sospecha clínica de posible rotura uterina.

\section{CONCLUSIONES}

El manejo multidiciplinario permite reducir el riesgo de mortalidad y morbilidad materna grave, en patología obstétrica con potencial hemorrágico grave, como son los casos de percretismo placentario severo.

\section{REFERENCIAS}

1. Zelop CM, Harlow BL, Frigoletto, et al. Emergency peripartum hysterectomy. Am J Obstet Gynecol 1993;168:1443-8.

2. Vedantham S, Goodwin SC, McLucas B, Mohr G. Uterine artery embolization: an underused method of controlling pelvic hemorrhage. Am J Obstet Gynecol 1997;176:938-48.

3. Sumigama S, Ikakura A, Ota $\mathrm{T}$, et al. Placenta increta/ percreta in Japan: a retrospective study of ultrasound findings, management and clinical course. J Obstet Gynecol Res 2007;33:606-11.

4. Belfort MA. Placenta accreta. Publications Committee, Society for Maternal-Fetal Medicine. Am J Obstet Gynecol 2010:203:430-9.

5. Bodner LJ, Nosher JL, Gribbin C, et al. Balloon-assisted occlusion of the internal iliac arteries in patients with placenta accreta/percreta. Cardiovasc Intervent Radiol 2006;29:354-61.

6. Tan $\mathrm{Ch} \mathrm{H}$, Tay $\mathrm{KH}$, Shea $\mathrm{K}$, et al. Perioperative endovascular internal iliac artery occlusion balloon placement in management of placenta accreta. AJR 2007; 189:1158-63.

7. Chou YJ, Cheng YF, Shen CC, et al. Failure of uterine arterial embolization: placenta accreta with profuse pospartun hemorrhage. Acta Obstet Gynecol Scand 2004;83:688-90.
8. Shirvastava V, Nageotte M, Major C, et al. Case control comparison of cesarean hysterectomy with and without prophylactic placement of intravascular balloon catheters for placenta accreta. Am J Obstet Gynecol 2007;197:402.e1-e5

9. Palacios JM, García R, Barbosa N, et al. Lower uterine blood supply: extrauterine anastomotic system and its application in surgical devascularization techniques. Acta Obstet Gynecol 2007;86:228-34.

10. Rohen JW, Yokochi Ch, Lütjen-Dreecool E. Atlas de anatomía humana: estudio fotográfico del cuerpo humano. 5 $5^{\text {a }}$ ed. Madrid: Elsevier; 2003.

11. Chait A, Molt A, Nelson JH. The colateral arterial circulation in the pelvis. AJR 1968;102:392-400.

12. Evans $S$, McShane $P$. The efficacy of internal iliac artery ligation in obstetric hemorrhage. Surg Gynecol Obstet 1985;160:250-3.

13. Comstock C, Love J, Bronsteen RA, et al. Sonographic detection of placenta accreta in the second and third trimesters of pregnancy. Am J Obstet Gynecol 2004;190:1135-40.

14. Yang JL, Lim YK, Kim KH, et al. Sonographic findings of placental lacune and the prediction of adherent placenta in women with placenta previa totalis and prior cesarean section. Ultrasound Obstet Gynecol 2006;28:178-82.

15. Shih JC, Liu KL, Shyu MK. Temporary balloon occlusion of the common iliac artery: new approach to bleeding control during cesarean hysterectomy for placenta percreta. Am J Obstet Gynecol 2005;193:1756-8.

16. Knölfler M. Critical growth factors and signaling pathways controlling human trophoblast invasion. Int $\mathrm{J}$ Dev Biol 2010;54:269-80.

17. Ahmed A, Dunk C, Ahmad S, Khaliq A. Regulation of placental vascular endotelial growth factor (VEGF) and placenta growth factor (PIGF) and soluble Flt-1 by oxygen-a-review. Placenta 2000;21(Suppl):S16-24.

18. Wehrum M, Buhimschi I, Salafia $C$, et al. Accreta complicating complete placenta previa is characterizes by reduced systemic levels of vascular endotelial growth factor and by epitelial-to-mesenchymal transition of the invasive trophoblast. Am J Obstet Gynecol;204:411.e1-11

19. Chou MM, Ke YM, Wu HC, et al. Temporary crossclamping of the infrarenal abdominal aorta during cesarean hysterectomy to control operative blood loss in placenta previaincreta/percreta. Taiwan $\mathrm{J}$ Obstet Gynecol 2010;49:72-6.

20. Doumouchstsis SK, Papageorghiou A, Arulkumuran S. Systematic review of conservative management of pospartum hemorrhage: what to do when medical treatment fails. Obstet Gynecol Survey 2007;62:540-7 